\title{
A New Wavelet-based Algorithm for R-peak Detection in ECG and it's a Comparison with the Currently Existing Algorithms
}

\author{
M. S. Dahwah \\ Ph.D. student at Applied Mathematics and \\ Informatics Department \\ Mari State University \\ Yoshkar-Ola, Russia
}

\author{
A. N. Leukhin \\ Professor at Applied Mathematics and Informatics \\ Department \\ Mari State University \\ Yoshkar-Ola, Russia
}

\begin{abstract}
The purpose of this paper is to develop a new approach for Rpeak detection in ECG and compare it with the most effective and existing algorithms. The proposed approach is based on DWT and envelope for the first stage of preprocessing and decision or detecting it was achieved by adaptive thresholding. The proposed algorithm is compared with Pan \& Tompkins, Savitzky-Golay smoothing filter, Hilbert and wavelet transforms as well as fast Fourier transform algorithm to investigate these techniques of $\mathrm{R}$ peak detection and evaluate the wavelet-based algorithm comparing with them. The algorithms are evaluated in the experimental section using ECG signals from the MIT-BIH database. The results of detection algorithms show that the proposed wavelet-based algorithm gets the highest sensitivity by $99.9 \%$ with higher reliability compared to other algorithms, also by analyzing the precision of them, it's come to light that FFT improved the highest precision with $99.7 \%$.
\end{abstract}

\section{Keywords}

ECG, Pan \& Tompkins, Savitzky-Golay smoothing filter, Fast Fourier transform, wavelet transforms, Hilbert transform, R peak.

\section{INTRODUCTION}

One of the most popular bio-signals in the biomedical engineering is electrocardiography, and $\mathrm{R}$ peak is the most important part of ECG signal because of it's an essential role in detection, segmentation and feature extraction in ECG and also in determining heart rate variability.

The detection of peaks and feature extraction in bio-signals are an important step in many processing and classification applications, so it is very important to have an accurate algorithm for detecting this peaks in several signals such ECG shown in Fig.1, as well as determine the exact time when it appears, and this will be possible by reducing noise as an initial step to be able for a high probability of correct detection $[1,2]$.

The preprocessing of ECG signal involves removing of baseline wanders and power line interference. Baseline wander is usually ranged between $0.5 \mathrm{~Hz}$ up to $0.75 \mathrm{~Hz}$ during the contraction of muscles. A diverse source of noise is because of the electromagnetic fields by the power line with the frequency of $50 \mathrm{~Hz}(60 \mathrm{~Hz}$ in some countries, i.e. US and Japan). Types of different noises make the detection of ECG peaks more complicated as the low amplitude waveforms become unreliable and spurious waveforms may be introduced [3].

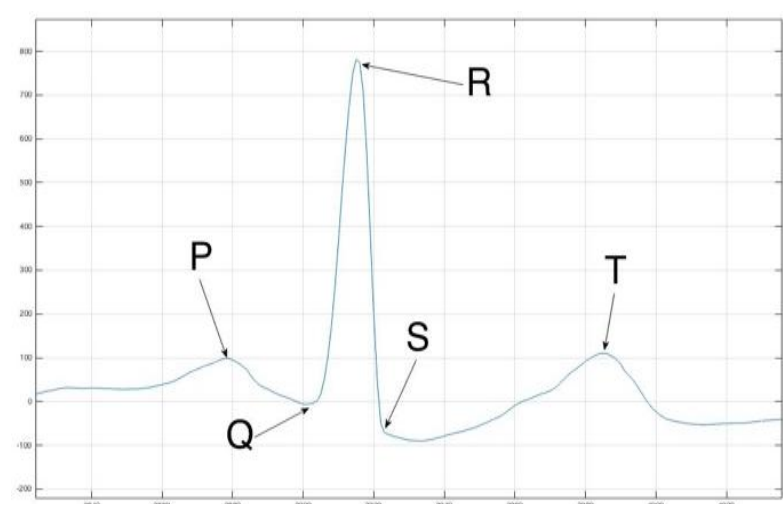

Fig 1: Sample ECG from MIT-BIH Database

The researchers, over the last 20 years, shows that the suggested algorithms in this paper for ECG analysis and specifically for peaks detection based on several signal processing techniques have reached a reliable detection performance [4].

In the field of ECG processing various algorithms have been reported specifically for the QRS complex such:

Differentiation algorithms [2]

Digital filters [5-9]

Neural networks [10-12]

In fact, most of the presented algorithms have a fundamental problem known as sensitivity to noise [13]. The problem of sensitivity to noise in ECG signal in itself is complex and because of that, in this paper, it is chosen six algorithms for preprocessing the electrocardiography in order to reduce noise before detecting $\mathrm{R}$ wave. This paper aims to get benefits from the advantages of Pan \& Tompkins algorithm, Savitzky-Golay smoothing filter, Hilbert and wavelet transform as well as fast Fourier transform in order to approach an optimum algorithm for $\mathrm{R}$ peak detection.

\section{MATERIALS AND ALGORITHMS}

In most peaks detection algorithms there are two varied stages, the first one is preprocessing and the second it will be the decision. In the preprocessing stage, different techniques are applied to the signal, six of these approaches were applied to ECG signal in this work. For better evaluation of the algorithms, they are tested on ECG signals selected from the MIT-BIH database with 9000 samples and results will be related to each algorithm that will be shown separately in each algorithm sections. 


\subsection{Pan \& Tompkins}

In this section, has been employed Pan \& Tompkins algorithm. This algorithm is based on passing ECG signal successively through some processes which based on pand-pass filter, differentiator and squaring[14]. The pand-pass filtering removes baseline wander and the high-frequency noise from the original ECG signal [15]. The differentiator preserves the information about the QRS complex slopes [16]. ECG squaring helps to emphasize the differences between the QRS complex with $\mathrm{P}$ and $\mathrm{T}$ waves [17].

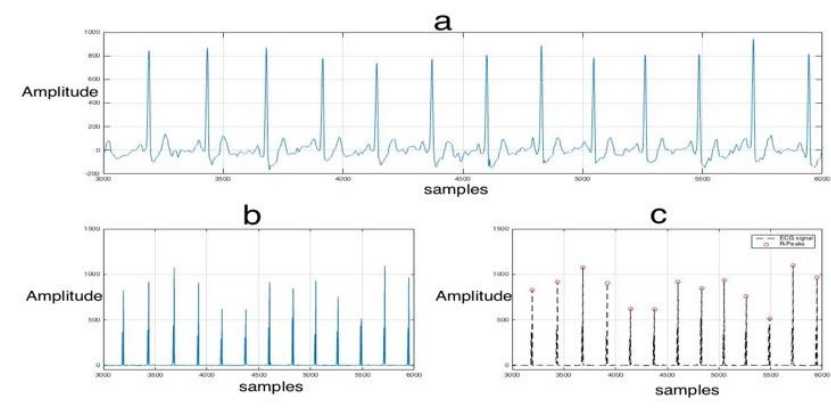

Fig 2: Sample ECG data, (a) original ECG, (b) processed ECG, (c) $R$ detected in ECG signal

Original ECG is shown in Figure 2 (a), in order to attenuate noise the ECG signal passes through a digital pand-pass filter composed of cascaded high and low pass filters, the output of this filter after the differentiation and squaring shown in (b), the final signal with the detected $\mathrm{R}$ peaks are presented in (c).

\subsection{Savitzky-Golay smoothing filter}

The main purpose of the Savitzky-Golay filter is to smooth the data, such filters are also named least squares or digital smoothing polynomial filters. In this section, smoothing ECG signal and then adaptive thresholding technique for $\mathrm{R}$ wave detection it was applied. According to Table 1, the algorithm which introduced is based on using three general stages including Savitsky-Golay filter, squaring and adaptive thresholding. Fig.3 shows ECG signals at various steps in signal processing by S-GSF algorithm.

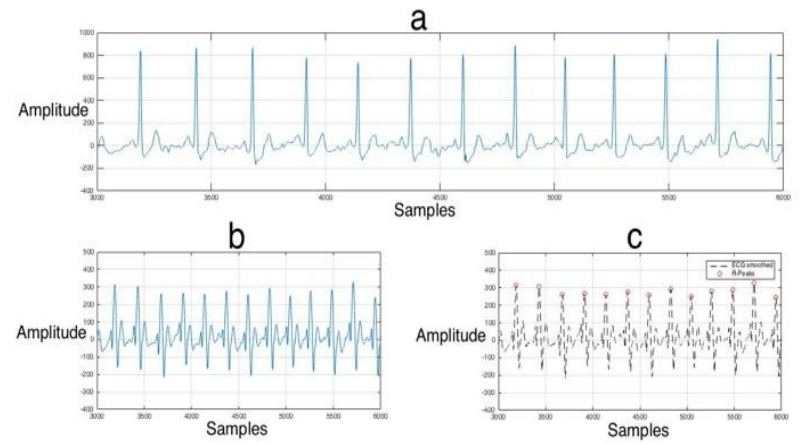

Fig 3: Sample ECG data (a), Original ECG: (b), processed ECG: (c), $R$ detected in ECG signal

\subsection{Wavelet transform}

The ability of the wavelet transform is tested in different works of peaks detection [18-20]. In this section, has been applied algorithm based on wavelet transform, is proposed for noise reduction and $\mathrm{R}$ peak detection. According to Table 1, WT algorithm has appeared a low probability of correct detection as well as the probability of false detection. The algorithm introduced in this section is based on two general stages: WT and adaptive thresholding. Figure 4 shows ECG signals at various steps in signal processing by WT algorithm.

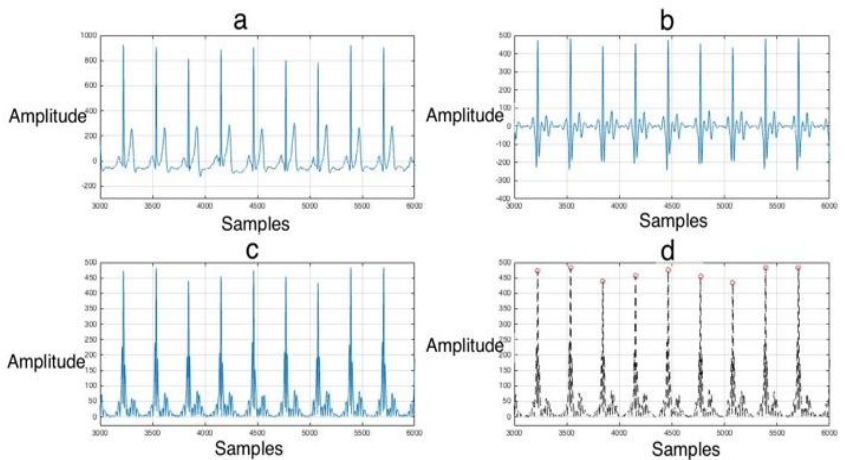

Fig 4: Sample ECG data, (a) Original ECG, (b) Reconstructed ECG, (c) abs Reconstructed ECG detection, (d) $R$ detected in ECG signal

\subsection{Hilbert transform}

Peak detection is one of the applications of the Hilbert transform using its properties. The algorithm uses ECG Hilbert transformed data in which undesirable peaks such as T and $\mathrm{P}$ waves are maximum minimized which makes $\mathrm{R}$ peak detection easily with the presence of various noises. According to Table 1, the algorithm introduced in this section is based on using two general stages including Hilbert transform and adaptive thresholding as shown in Fig.5.

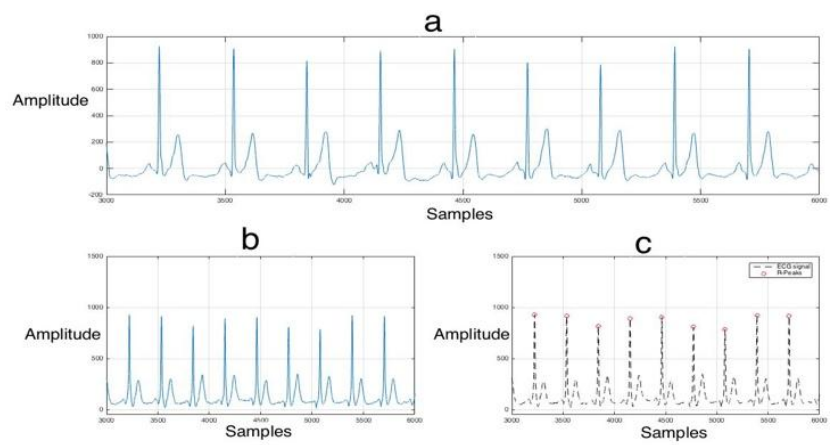

Fig 5: Sample ECG data, (a) Original ECG, (b) Filtered ECG, (c) R peaks detection

\subsection{Fast Fourier Transform}

The main idea of this algorithm is to apply direct fast Fourier transform, remove low frequencies and restore ECG with the help of inverse fast Fourier transform and then adaptive thresholding technique for $\mathrm{R}$ wave detection.

The noisy ECG is shown in Figure 6 (a), after removing the low frequencies from the signal, the output of this step shown in (b), the final signal with the detected $\mathrm{R}$ peaks are presented in (c).

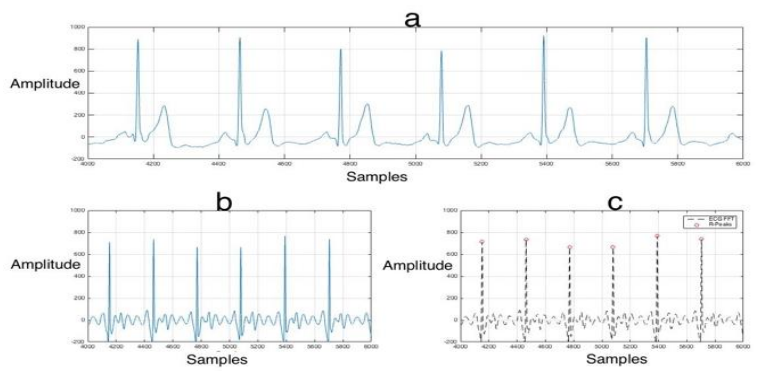

Fig 6: Sample ECG data, (a) Original ECG, (b) Filtered ECG, (c) $R$ detected in ECG signal 


\subsection{A new wavelet-based algorithm}

In this work, it was developed an algorithm for detection $\mathrm{R}$ peaks of ECG signals. It reliably detects $\mathrm{R}$ peaks based upon DWT and envelope for the first stage of preprocessing and decision or detecting it was achieved by adaptive thresholding. The wavelet transform can be used as a signal decomposition in the plane of the time scale. There are many applications of wavelet transforms, such as compression of sub-band encoding data, detection of characteristic points, and noise cancellation. To reduce the noise of the ECG signal, many algorithms are available, such as digital filters FIR or IIR, adaptive algorithm, and wavelet transformation thresholding algorithms [21].

The wavelet transform describes a decomposition process with several resolutions in terms of a signal extension to a set of wavelet-basis functions. The wavelet has its excellent spatial frequency localization property. The use of DWT in a 1D signal corresponds to a 1D filter in each measurement [22]. The threshold value is used in the frequency domain to smooth out or to remove certain coefficients of the wavelet signal subsignals of the measured signal. The noise scale in the signal is effectively reduced in a non-stationary environment. The algorithm of noise reduction, which applies a threshold value in the frequency domain, is proposed by Donoho [23].

The introduced algorithm is about decomposition and reconstruction the ECG signal into five scales of wavelets by using Daubechies family and getting a threshold value where where the error level is minimized between the thresholded noisy and the original signal.

The algorithm can be sequenced into this steps: 1) Decomposing of signals using wavelet transform: The signals are decomposed into five levels by discrete wavelet transform using wavelet (db4); 2) Choosing threshold value; 3) Reconstruction: The signal is reconstructed using the IDWT [21]. After DW reconstruction of ECG, the signal is squared point by point. This makes all data points positive and does nonlinear amplification of the output of the ECG signal. After that, the peak detector is used to find the peaks in the ECG signal envelope by adaptive thresholding.

Fig.7 shows signals at various steps in digital signal processing (a) show the original ECG. In order to attenuate noise, the ECG signal was decomposed and reconstructed in (b) after that squaring the ECG signal is presented in (c) also (d) shows ECG signal envelope as (e) shows the final signal with $R$ peak detected.

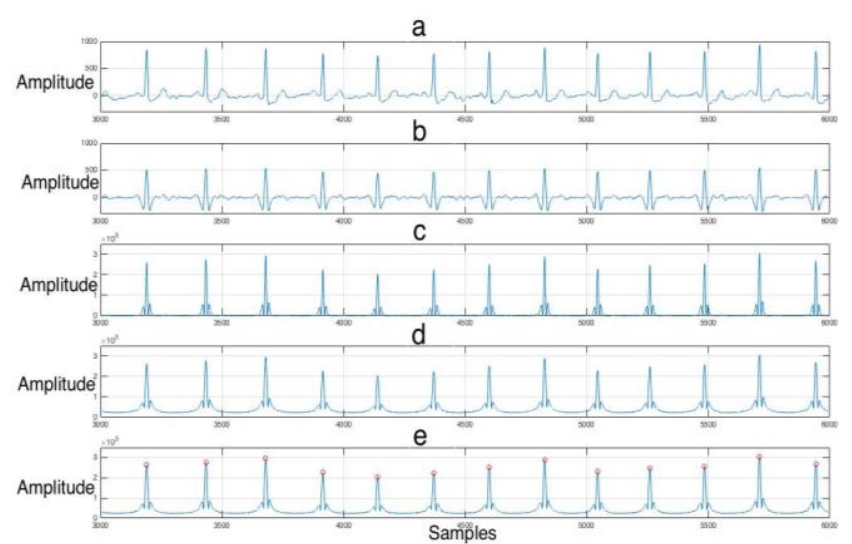

Fig 7: The steps of the wavelet-based algorithm in ECG

processing and $\mathbf{R}$ detection

\section{EVALUATION AND COMPARISON}

The evaluation of the performance of the algorithms. Depends on two parameters which should be used to evaluate the detection performance; that is,

$\begin{array}{ll}\mathrm{TPR}=\mathrm{TP} / \mathrm{P} & \text { sensitivity } \\ \mathrm{PPV}=\mathrm{TP} / \mathrm{TP}+\mathrm{FP} & \text { precision }\end{array}$

where TP denotes the number of true positive detections, $\mathrm{P}$ positive samples, and FP the number of false positives. Furthermore, to achieve comparable and reproducible results, the evaluation needs to be carried out on standard databases which are MIT-BIH in this research. Variety of $\mathrm{R}$ peak detection algorithms presented in table 1 reflects the need for a reliable R peak detection in ECG processing. Sensitivities of about $99.9 \%$ are possible for $\mathrm{R}$ peak detectors as shown in table 1 .

Table 1. Statistical results for the algorithms

\begin{tabular}{|c|c|c|c|c|c|}
\hline & Algorithm & $\begin{array}{c}\text { QRS detection } \\
\text { sequence }\end{array}$ & Efficiency & TPR & PPV \\
\hline 1 & $\begin{array}{c}\text { Pan And } \\
\text { Tomp } \\
\text { kins }\end{array}$ & $\begin{array}{c}\text { First derivative } \\
+ \text { squaring } \\
+ \text { pand- } \\
\text { pass filter } \\
+ \text { adaptive } \\
\text { thresholdi } \\
\text { ng }\end{array}$ & Medium & 98.9 & 98.2 \\
\hline 2 & $\begin{array}{c}\text { Savitzky- } \\
\text { Golay } \\
\text { smoot } \\
\text { hing } \\
\text { filter }\end{array}$ & $\begin{array}{c}\text { Savitsky-Golay } \\
\text { filter } \\
\text { +squaring } \\
\text { +adaptive } \\
\text { thresholdi } \\
\text { ng }\end{array}$ & Low & 95.8 & 97.7 \\
\hline 3 & $\begin{array}{c}\text { Wavelet } \\
\text { transf } \\
\text { orm }\end{array}$ & $\begin{array}{c}\text { WT+ adaptive } \\
\text { thresholdi } \\
\text { ng }\end{array}$ & Low & 95.9 & 97.4 \\
\hline 4 & $\begin{array}{l}\text { Hilbert } \\
\text { transf } \\
\text { orm }\end{array}$ & $\begin{array}{l}\text { Hilbert } \\
\text { transform } \\
\text { + adaptive } \\
\text { thresholdi } \\
\text { ng }\end{array}$ & Low & 97,5 & 96.7 \\
\hline 5 & $\begin{array}{c}\text { Fast } \\
\text { Fouri } \\
\text { er } \\
\text { transf } \\
\text { orm }\end{array}$ & $\begin{array}{c}\text { FFT/IFFT+ } \\
\text { adaptive } \\
\text { thresholdi } \\
\text { ng }\end{array}$ & Low & 97.2 & 99.7 \\
\hline 6 & $\begin{array}{c}\text { A new- } \\
\text { wavel } \\
\text { et } \\
\text { based } \\
\text { algori } \\
\text { thm }\end{array}$ & $\begin{array}{c}\text { Wavelet } \\
\text { decompos } \\
\text { e and } \\
\text { reconstruc } \\
\text { tion, + } \\
\text { squaring+ } \\
\text { envelope, } \\
\text { + adaptive } \\
\text { thresholdi } \\
\text { ng }\end{array}$ & High & 99.9 & 99.3 \\
\hline
\end{tabular}




\section{RESULTS AND DISCUSSION}

About 725 beats from the MIT-BIH Arrhythmia database, without exception, were used for evaluating the algorithms. The results are presented in Table 1. Two parameters were used to evaluate the algorithms for each signal; that is, sensitivity and precision. From the chart and Table 1 it's come to light that a new wavelet-based algorithm improved the highest sensitivity by $99.9 \%$, with precision by $99.3 \%$ as shown in fig.8. The evaluation data of all algorithms presented in this work in Table 1 are shown as a chart in Fig.8.

$\mathrm{R}$ peak is detected using a new wavelet-based algorithm with high reliability. These detection rates may be sufficient for diagnostic applications in cardiac therapy, as well as a higher performance may be necessary for research purposes also.

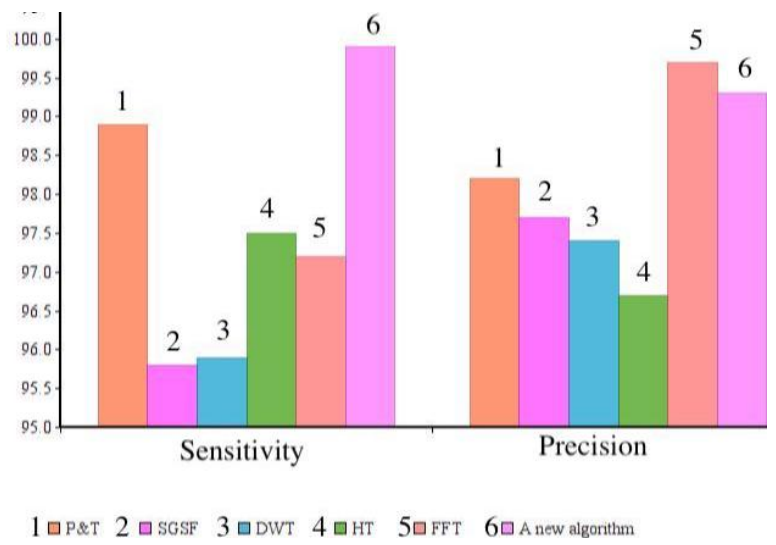

Fig 8: A chart of the evaluation data of all $R$ detection algorithms

\section{REFERENCES}

[1] M.A. Khayer, M.A.Haque., 2004 ECG Peak Detection using Wavelet Transform. International Conference on Electrical Computer Engineering, 518-521.

[2] J. Fraden, M.R. Neuman., 1980 QRS wave detection. Medical and Biological Engineering and Computing, 125-132.

[3] J. Pan, W. J. Tompkins., 1985 A Real-Time QRS Detection Algorithm. IEEE Transactions on Biomedical Engineering, 230-236.

[4] Francois Portet, Guy Carrault., 2005 Piloting real-time QRS detection algorithms in variable contexts. European Medical \& Biological Engineering Conference,1-7.

[5] Okada M., 1979 A digital filter for the QRS complex detection. IEEE Trans. Biomed. Eng.,700-703.

[6] Engelse WA, Zeelenberg C., 1979 A single scan algorithm for QRS detection and feature extraction. IEEE Comput Cardiology, 37-42.

[7] Hamilton PS, Tompkins WJ., 1986 Quantitative investigation of QRS detection rules using the MIT/BIH arrhythmia database. IEEE Trans. Biomed. Eng., 57-65.

[8] Keselbrener L et al., 1997 Nonlinear high pass filter for R-wave detection in ECG signal. Med. Eng. Phys., 738741.

[9] Suppappola S, Sun Y., 1994 Nonlinear transforms of ECG signals for digital QRS detection: A quantitative analysis. IEEE Trans. Biomed. Eng., 397-400.
[10] Dokur Z, Olmez T, Yazgan E, Ersoy OK., 1997 Detection of ECG waveforms by neural networks. Med. Eng. Phys., 738-741.

[11] Barro S, Fernandez-Delgado M, Vila-Sobrino JA, Regueiro CV, Sanchez E., 1998 Classifying multichannel ECG patterns with an adaptive neural network. IEEE Eng. Med. Biol. Mag., 45-55.

[12] Fernandez-Delgado M, Barro Ameneiro S., 1998 A multichannel ART-based neural network. IEEE Trans Neural Netw., 139-150.

[13] Hossein Rabbani, M. Parsa Mahjoob, E. Farahabadi, A. Farahabadi., 2011 R Peak Detection in Electrocardiogram Signal Based on an Optimal Combination of Wavelet Transform, Hilbert Transform, and Adaptive Thresholding. Journal of Medical Signals and Sensors, 91-98.

[14] L. Sathyapriya, L. Murali, T. Manigandan., 2014 Analysis and Detection R-Peak Detection using Modified Pan-Tompkins Algorithm. IEEE International Conference on Advanced Communication Control and Computing Technologies, 483-487.

[15] M. L. Ahlstrom, W. J. Tompkins., 2007 Digital Filters for Real-Time ECG Signal Processing Using Microprocessors. IEEE Transaction on Biomedical Engineering, 708-713.

[16] N. Arzeno, Z. Deng, and C. Poon., 2008 Analysis of First Derivative Based QRS Detection Algorithms. IEEE Transactions on Biomedical Engineering, 478-484.

[17] Kohler BU, Hennig C, Orlgmeister R., 2002 The principles of software QRS detection. IEEE Engineering in Medicine and Biology Magazine, 42-57.

[18] Abibullaev B, Seo H., 2011 A new QRS detection method using wavelets and artificial neural networks. Journal of Medical Systems, 683-691.

[19] Chen SW, Chen HC, Chan HL., 2006 A real-time QRS detection method based on moving-averaging incorporating with wavelet denoising. Computer Methods and Programs in Biomedicine, 187-195.

[20] Zidelmal Z, Amirou A, Adnane M, Belouchrani A., 2012 QRS detection based on wavelet coefficients. Computer Methods and Programs in Biomedicine, 490-496.

[21] Mikhled Alfaouri and Khaled Daqrouq., 2008 ECG Signal Denoising By Wavelet Transform Thresholding. American Journal of Applied Sciences, 276-281.

[22] Biswas, Anamitra Bardhan Roy, Nilanjan Dey., 2012 Wavelet-based QRS Complex Detection of ECG Signal. International Journal of Engineering Research and Applications, 2361-2365.

[23] D. L. Donoho., 1995 De-noising by soft thresholding.IEEE Transaction on Information Theory, 613-627.

[24] Addison, P. S., 2010 The illustrated wavelet transform handbook: introductory theory and applications in science, engineering, medicine and finance. CRC Press, Philadelphia. 